Published in final edited form as:

Personal Disord. 2012 July ; 3(3): 273-282. doi:10.1037/a0027331.

\title{
Emotional Processing in Borderline Personality Disorder
}

\author{
Michael K. Suvak ${ }^{1}$, Christopher T. Sege ${ }^{2}$, Denise M. Sloan ${ }^{3}$, M. Tracie Shea ${ }^{4}$, Shirley Yen ${ }^{5}$, \\ and Brett T. Litz ${ }^{3}$ \\ 1VA National Center for PTSD, VA Boston Healthcare System and Suffolk University \\ 2VA National Center for PTSD, VA Boston Healthcare System \\ ${ }^{3}$ VA National Center for PTSD, Boston Healthcare System and Boston University School of \\ Medicine \\ ${ }^{4}$ Veterans Affairs Medical Center, Providence, Department of Psychiatry \& Human Behavior, \\ Brown Medical School \\ ${ }^{5}$ Department of Psychiatry \& Human Behavior, Brown Medical School
}

\begin{abstract}
This study examined whether individuals with borderline personality disorder (BPD) would exhibit augmented emotional responses to picture stimuli after being challenged with an ideographic interpersonal conflict script. Participants were 24 adults diagnosed with BPD, 23 adults diagnosed with obsessive compulsive personality disorder (OCPD), and 28 normal controls. Participants viewed emotionally evocative pictures before and after listening to the interpersonal script while a variety of physiological measures were recorded. Findings indicated that the interpersonal script was effective in eliciting enduring emotional responses from the BPD group relative to the control groups. However, despite the effectiveness of the interpersonal challenge task, there were no group differences in emotional responding to the affect eliciting stimuli. The findings underscore the complexities involved in examining emotional dysregulation in BPD in a laboratory setting.
\end{abstract}

\section{Keywords}

Borderline personality disorder; emotion responding; startle reflex; psychophysiology; heart rate; electromyography; skin conductance

\begin{abstract}
Difficulty in processing and managing emotions is viewed by some as the core feature of borderline personality disorder (BPD; e.g., Linehan 1993). The DSM-IV (American Psychiatric Association [APA], 1994) criteria of intense anger and affective instability explicitly identify emotion regulation problems, whereas other problems (e.g., frantic efforts to avoid abandonment, repeated suicide attempts or gestures, intense and unstable relationships) may be viewed as strategies to cope with or consequences of this core deficit.
\end{abstract}

Address correspondence to Denise Sloan, Ph.D., National Center for PTSD (116B-2), VA Boston Healthcare System, $150 \mathrm{~S}$. Huntington Avenue, Boston, MA 02130; Denise.Sloan@va.gov.. 
Linehan (1993) posited that patients with BPD are hyper-sensitive to emotional challenges and poorly manage their subsequent reactions, resulting in conflict and dysregulation.

Despite the centrality of emotion to the concept of BPD, surprisingly little research has directly examined emotional responding in individuals with BPD (for a review see, Rosenthal et al., 2008). One exception is a series of studies by Herpertz and colleagues, which investigated physiological indicators of emotional reactions to standardized emotioneliciting pictures (Herpertz, Kunert, Schwenger, \& Sass, 1999; Herpertz et al., 2000; Herpertz et al., 2001). In these studies, participants with BPD did not show more intense responses to any of the affect evoking pictures, failing to support the hypothesis of emotional hyper-responsiveness in BPD.

Hazlett et al. (2007) examined eye-blink startle in response to unpleasant, BPD-relevant words (e.g., hate), and neutral words in a BDP group compared to a healthy control group. Participants with BPD exhibited larger startle responses while processing unpleasant words in comparison to the healthy control group; no group differences were found while processing neutral words. However, as only BPD-relevant unpleasant words were presented, it is unclear whether the startle potentiation exhibited by the BPD group was due to the fact that the words tapped BPD-relevant themes or that they were merely unpleasant. In addition, both the BPD and healthy control groups rated the unpleasant words as more arousing than the neutral pictures. Consequently, it is unclear whether the results are due to differences in the valence or arousal value of the stimuli.

The empirical evidence to date suggests that there may be limits, or contextual constraints, to emotional abnormalities in BPD. In other words, emotional-processing abnormalities may arise in some contexts and not others. One possibility is that individuals with BPD exhibit emotional hyper-reactivity after being provoked or triggered by conflict-laden interpersonal challenges. For example, emotional-processing abnormalities in BPD may be secondary to episodes of perceived violation or perceived withdrawal of love, which results in anger or rage, characterized by high arousal and cognitive and behavioral disinhibition (e.g., Hochhausen, Lorenz, \& Newman, 2002). High arousal angry states in BPD would in turn alter appraisal of emotional cues and interfere with otherwise intact emotional capabilities. Ebner-Priemer and colleagues (2008) found support for this hypothesis, such that BPD individuals displayed greater inability to label emotions during periods of psychological distress.

Emotional-processing problems secondary to contexts that trigger maladaptive schemas in BPD is also consistent with Beck's cognitive conceptualization of personality disorders, which posits that when particular schemas are triggered, the threshold for emotional activation is low and readily triggered by a trivial stimulus (Beck \& Freeman, 1990). This suggests that contexts or events that prime or trigger BPD-relevant schemas would lead to subsequent abnormal emotional responses.

The primary aim of this study was to investigate the emotional-processing consequences of exposure to a personally relevant challenge in BPD. Participants included individuals diagnosed with $\mathrm{BPD}$, individuals diagnosed with obsessive compulsive personality disorder 
(OCPD), and a normal control (NC) group. We used an "extreme group" approach so that any observed differences in the BPD group could be attributable to BPD symptoms rather than personality disorder more generally. We elected to use the OCPD group because symptoms of this personality disorder are highly distinct from BPD.

We predicted that, at baseline, there would be normative patterns of psychophysiological response to pleasant, unpleasant, and neutral images in the BPD group, which would not differ from OCPD and NC groups. However, after exposure to an ideographic violationrelated challenge or prime, we expected that participants with BPD would be less responsive to pleasant images and more responsive to unpleasant images, relative to their baseline responses, and relative to $\mathrm{NC}$ and the OCPD groups.

\section{Method}

\section{Participants}

Participants were 75 adult females ( $n=24,23$, and 28 in the BPD, OCPD, and NC groups, respectively) with a mean age of $32.89(S D=12.19)$ and mean years of education of 15.35 $(S D=2.23)$.The majority $(77.4 \%)$ were Caucasian. Participants were informed that the purpose of the study was to determine how personality affects emotions. Financial compensation was provided for their time and travel expenses. The study was approved by the local Institutional Review Boards.

Most participants were recruited using flyers posted on community and internet bulletin boards. Five participants from the BPD group and five from the OCPD group were recruited from the Brown University and McLean Hospital sites of the Collaborative Longitudinal Study of Personality Disorders (CLPS, Zanarini et al., 2000). For the current study, BPD participants could not endorse OCPD diagnostic symptoms, and participants with OCPD could not endorse BPD diagnostic symptoms. Participants in the NC group could not meet lifetime or current diagnostic criteria for any Axis-I or Axis-II disorders. Exclusion criteria for all participants included lifetime psychosis diagnosis and self-reported hearing problems. All participants had to be at least 18 years old and able to provide informed consent. A substantial proportion of BPD and OCPD participants reported current psychotropic medication use (58\% and $42 \%$, respectively); none of the NC participants reported medication use.

\section{Stimulus Materials}

Pleasant, unpleasant, and neutral images were selected from the International Affective Picture System (IAPS; Lang, Bradley, \& Cuthbert, 2005) on the basis of normative ratings for females. Pictures were displayed four feet from the participant on a 27.5 (width) x 15.5 (height) inch computer monitor. Pictures were presented for $6 \mathrm{~s}$ with inter-trial intervals (ITIs) ranging from 18-26 s. Consistent with procedures used by other investigators (e.g., Bradley, Codispoti, Cuthbert, \& Lang, 2001), participants viewed a total of 60 images across two blocks; each block contained 10 pleasant, 10 unpleasant, and 10 neutral images. Only responses to 36 images (six pleasant, six neutral, six unpleasant per block) during which startle probes were presented are included in the current study. The acoustic startle probe 
consisted of a 50-ms burst of $104 \mathrm{~dB}$ white noise with immediate $(10 \mathrm{~ms})$ rise time. The startle stimulus was presented binaurally through Maxwell NC-II headphones.

\section{Measures}

The Diagnostic Interview for Personality Disorders (DIPD; Zanarini.,Frankenburg, Sickel, \& Yong, 1996) BPD and OCPD modules were the primary diagnostic instruments used in this study. This instrument assesses symptoms consistent with DSM-IV symptoms of BPD and OCPD. Symptoms must be present and pervasive for at least two years and characteristic of the person during her adult life. The Structured Clinical Interview for the DSM-IV Axis I Disorders (SCID-I; First, Spitzer, Gibbon, \& Williams, 1996) was used to identify Axis-I pathology in the study sample. The Longitudinal Interval Follow-up Evaluation (LIFE; Keller, Lavori, Friedman, \& Nielsen, 1987) was used to assess the presence of psychotic symptoms.

All diagnostic instruments were administered either by CLPS licensed clinicians or by postdoctoral fellows or advanced doctoral-level psychology students. All assessments were supervised and checked for diagnostic accuracy by an experienced doctoral-level psychologist.

Interpersonal violation script-A semi-structured interview was used to elicit a narrative of an anger-provoking interpersonal conflict with someone close to the participant. A $90 \mathrm{~s}$ narrative script was then generated from the interview. The method for generating the script followed the procedure developed by Lang and colleagues (e.g., Lang \& Cuthbert, 1984). Following presentation of the script, the participant was asked to close her eyes and imagine the event taking place in real-time.

\section{Emotion Response Measures}

A computerized version of the Self-Assessment Manikin (SAM; Bradley \& Lang, 1994) was used to assess self-reported changes in affect during the laboratory session. The SAM uses manikin figures on a 9-point scale to represent two affect dimensions (i.e., valence and arousal). In addition, participants reported on their experience of anger during the laboratory procedure using a computerized 9-point Likert-type scale.

\section{Apparatus and Psychophysiological Measures}

Labtech Notebook Pro software (Labtech Corporation, 2000) and a Coulbourn (Allentown, PA) amplifier stack were used to collect psychophysiological data. SuperLab software (Cedrus Corporation, 1999) using a 1000-Hz timer was used to display digital images, collect ratings data, coordinate the onset and offset of data collection, and control the timing of all experimental stimuli.

Startle response-The eyeblink component of the startle reflex was measured by recording electromyography (EMG) activity from Beckman miniature $\mathrm{Ag} / \mathrm{AgCl}$ electrodes positioned over the orbicularis oculi muscle beneath the left eye. Startle probes were presented at 3.5, 4.5, or $5.5 \mathrm{~s}$ after picture onset (counterbalanced with valence) and 9 additional probes were presented during intervals in which no picture was present, for a total 
of 39 probes per block. Three of the no-picture probes occurred during the $90 \mathrm{~s}$ interval prior to the first picture trial. The remaining probes were interspersed at unpredictable intervals across each block. Startle data for five participants (1 BPD, 2 OCPD, 2 NC) were removed because of excessive artifact or equipment malfunctions.

Heart rate (HR) - HR activity was recorded with $1 \mathrm{~cm}$ Beckman $\mathrm{Ag} / \mathrm{AgCl}$ electrodes positioned on the right and left inner forearms. Interbeat intervals were recorded in $\mathrm{ms}$ and reduced online to HR in beats per minute (BPM) in $0.5 \mathrm{~s}$ intervals. Change scores for the picture processing procedure were computed by subtracting the mean of the $6 \mathrm{~s}$ baseline interval from the minimum value during the first $3 \mathrm{~s}$ of picture viewing (deceleration) and from the maximum value during the last $3 \mathrm{~s}$ of picture viewing (acceleration). For script and imagery periods, analyses compared the mean of the $60 \mathrm{~s}$ baseline interval prior to the script with the mean of the maximum $10 \mathrm{~s}$ interval during the $90 \mathrm{~s}$ script period and $60 \mathrm{~s}$ imagery period. HR data from 15 participants (4 BPD, $4 \mathrm{OCPD}, 7 \mathrm{NC}$ ) were removed because of excessive artifact or technical malfunctions.

Skin conductance (SC) - SC was recorded from adjacent sites on the hypothenar eminence of the non-dominant hand with 1-cm Beckman $\mathrm{Ag} / \mathrm{AgCl}$ electrodes filled with Unibase-saline paste. During the IAPS blocks, sampling $(10 \mathrm{~Hz})$ began $1 \mathrm{~s}$ prior to the onset of the image and continued through the $6 \mathrm{~s}$ presentation interval. Change in SC level (SCL) during picture processing was computed by subtracting the mean of the $1 \mathrm{~s}$ baseline interval prior to the onset of the picture from the mean of the $6 \mathrm{~s}$ picture-viewing interval. Change in SCL during the script and imagery periods was computed by subtracting the mean of the 60 $\mathrm{s}$ baseline from the mean of the maximum $10 \mathrm{~s}$ interval during each period. SC data from five participants (3 BPD, 2 OCPD) were removed because of excessive artifact.

Facial EMG-Corrugator and zygomatic facial EMG activity was recorded from Beckman miniature $\mathrm{Ag} / \mathrm{AgCl}$ electrodes filled with electrolyte paste positioned according to standard guidelines (Fridlund \& Cacioppo, 1986). EMG during picture processing was sampled (10 $\mathrm{Hz}$ ) beginning $1 \mathrm{~s}$ prior to picture onset through the $6 \mathrm{~s}$ picture viewing period. Facial EMG activity in response to the IAPS pictures and to the script-imagery procedure was computed in the same manner as SC. Corrugator EMG data were removed for three participants (2 BPD, 1 OCPD) and zygomatic EMG data were removed for two participants (1 BPD, 1 OCPD) due to equipment malfunctions.

\section{Procedure}

The study was conducted over two sessions scheduled approximately one week apart. Upon arrival for the first session, participants were informed about the nature and requirements of the study, provided consent, and completed the diagnostic interviews.

At the second session, participants were seated in a recliner in a dimly lit, sound-attenuated room and physiological sensors were applied. A computer mouse was located on the right arm of the chair. The participant was provided instructions for completion of the rating scales and was administered a baseline SAM ratings. Following a $90 \mathrm{~s}$ baseline recording period, the participant viewed the first block of IAPS images. Each image was followed by a 
$6 \mathrm{~s}$ blank screen and then presentation of SAM ratings. Post-baseline SAM ratings were administered, along with an anger rating to assess levels of anger before the script-imagery procedure. This was followed by the presentation of the anger script and the imagery period, and then another set of SAM and anger ratings.

\section{Data Analysis Plan}

For the psychophysiological data, mixed-effects regression analyses were conducted using the Hierarchical Linear and Non-Linear Modeling Software Program (HLM6; Raudenbush, Bryk, \& Congdon, 2005). Dummy coded variables were used to analyze the categorical factors (valence, group, and condition). Primary analyses involved a 3 (Valence: Unpleasant, Neutral, Pleasant) x 3 (Group: BPD+, OCPD, NC) x 2 (Block: Baseline, Post-script) repeated-measures comparison. For each outcome, the first model evaluated all main effects, and the second and third models evaluated two-way and three-way interactions, respectively. Overall model fit was assessed by evaluating change in the deviance statistic ( $\Delta \mathrm{dev}$; which follows a chi-squared distribution; Raudenbush \& Bryk, 2002).

We used a model-comparison approach to examine specific main effects and interactions. Each factor of interest was removed sequentially, the model was recomputed, and a decrement in model fit as assessed by $\Delta \mathrm{dev}$. It is typical to test each individual main effect when controlling for all other main effects, each individual two-way interaction when controlling for all other two-way interactions, and so on. Regression coefficients were used to depict the nature of significant effects. This approach is analogous to examining the omnibus F-test in ANOVA and conducting specific contrasts to elucidate that nature of significant results. For estimates of within-subject effect sizes, we report the partial correlation coefficients ( $p r)$ for each coefficient. Kirk (1996) suggests .10, .24, and .37 for small, medium, and large effect sizes, respectively. For estimates of between-groups effect sizes, we report Cohen's d $(d)$ and use descriptors of effect size offered by Cohen (1988): d $=; .25$ small, .50 medium, and .80 large.

\section{Results}

A series of ANOVA and Chi Square analyses confirmed no significant group differences in demographic characteristics (average age, years of education and racial. A greater frequency of BPD participants met diagnostic criteria for depression disorders, panic disorder, and social anxiety disorder $(13,8$, and 9 , respectively) relative to the OCPD group $(5,4$, and 4 , respectively). Posttraumatic stress disorder was comparable in the two personality disorder groups (2 BPD and 3 OCPD participants).

\section{Manipulation check}

Mixed-effect regression with phase (pre-script, post-script, end of experiment) analyzed using dummy coded variables (producing a model analogous to a repeated-measures ANOVA) was conducted to assess the impact of the state manipulation on self-reports of affect. Summary statistics are displayed Table 1. Overall, BPD participants and OCPD participants reported significantly higher levels of arousal than NC participants. BPD participants also reported significantly higher levels of anger than both OCPD and NC 
participants. However, no significant Group x Phase two-way interactions emerged, indicating that the script did not differentially impact the three groups. The findings support successful manipulation of the script.

\section{Emotional Processing of Emotion-Eliciting Images}

The analyses were conducted in a similar manner for all outcomes, with one exception. Prior to testing primary hypotheses regarding emotion modulation of the startle response, growth curve analyses examined responses to startles presented when no images were displayed (, i.e., ITI startles) to evaluate initial startle response, habituation, and how these processes might differ as a function of group. As expected, a strong non-linear (i.e., natural log) habituation pattern emerged $(b=-.98, t=-4.19, p<.001, p r=.45)$ that accounted for $12 \%$ of the within-subjects variance in startle magnitude. Group did not significantly predict either initial status or the habituation effect. However, when analyzing the emotion modulation of the startle response during picture presentation, the natural log transformed startle serial number was included as a time varying covariate to statistically control for habituation.

Summary statistics for picture-processing variables as well as the significant test for each main effect, two-way interaction, and the three-way interaction can be found in Table 2. Importantly, the psychophysiological findings obtained in this study are consistent with the emotion picture processing literature (e.g., Bradley et al., 2001). Specifically, significantly greater startle reflex was observed during viewing of unpleasant images relative to neutral $(p r=.34)$ and pleasant images $(p r=.49)$. In turn, startle reflex was significantly greater while viewing neutral images relative to pleasant images $(p r=.21)$. Participants displayed significantly greater zygomatic facial EMG activity while viewing pleasant images compared to neutral $(p r=.20)$ and unpleasant images $(p r=.19)$, whereas greater corrugator facial EMG activity occurred while viewing unpleasant images relative to pleasant $(p r=.49)$ and neutral $(p r=.40)$ pictures. Greater SC activity occurred while viewing pleasant ( $p r=$. $41)$ and unpleasant pictures relative to neutral pictures, whereas SC $(p r=.34)$ activity did not differ between pleasant and unpleasant images $(p r=.15)$.

Significant group effects emerged for only two variables, self-reported arousal and HR-acc. For self-reported arousal, a significant Group X Block interaction emerged $(\Delta \mathrm{dev}=14.65, d f$ $=2, p<.001$ ). Examination of the regression coefficients revealed that during block 1 , participants in the OCPD group rated all images as more arousing than the $\mathrm{NC}\left(b_{\text {ocpd-nc }}=\right.$. $88, t=2.31, p<.05, d=.54)$ and the BPD groups $\left(b_{o c p d-n c}=.84, t=2.07, p<.05, d=.54\right)$; the difference between the NC and BPD groups was not significant. During block 2, there were no significant group differences ( $d$ 's ranging from .01 to .06 ).

For HR-acc, a significant Group X Valence two-way interaction emerged $(\Delta \operatorname{dev}=9.47, d f=$ $4, p<.05)$. Examining regression coefficients revealed that the pleasant-neutral and unpleasant-neutral contrasts were different for the BPD group compared to the OCPD ( $d$ for the BPD-OCPD difference in pleasant-neutral contrast $=.66$, unpleasant-neutral contrast $=$. 57 ) and NC groups ( $d$ for the BPD-NC difference in pleasant-neutral contrast $=.66$, unpleasant-neutral contrast $=.76$ ). For the BPD group, there were no statistically significant differences across the valence conditions. On the other hand, for the OCPD and NC groups, 
neutral images were associated with the largest HR accelerations. Furthermore, the neutralunpleasant contrast were statistically significant for both the NC and OCPD groups $(p<.01$ for the NC group and $p<.05$ for the OCPD condition), while the neutral-pleasant contrast approached statistical significance for both groups $(p<.07$ for the NC group and $p<.08$ for the OCPD condition). No statistically significant difference emerged in HR acceleration between the NC and OCPD conditions ( $d$ 's ranging from .01 to .27).

\section{Discussion}

Consistent with some prior research (e.g., Herpertz et al., 2001) the current study found that BPD individuals do not display or report emotional behavior abnormalities in highly controlled laboratory conditions. Although the interpersonal violation script was effective in eliciting arousal and anger, contrary to our prediction, the interpersonal violation script did not differentially impact the BPD group's emotional responding to emotionally evocative pictures, which is surprising given that emotion dysregulation is considered a core feature of BPD.

These null findings could be the result of many factors. First, because findings replicated the Herpertz et al. studies $(1999,2000,2001)$ using similar methods, one possible explanation is that the stimulus set was not sufficiently evocative. Static images that depict emotional scenes may not be sufficiently evocative to elicit emotional abnormalities in personality disorders. It is also possible that the picture processing interval of six seconds was too short to reveal differential responding. Lastly, we may have found group differences in emotional responding if we only used anger eliciting stimuli, which would have matched the anger prime used in this study.

On the other hand, it is plausible that picture stimuli used in our study, and used by Herpertz and colleagues, did not sufficiently tap BPD-relevant themes. Our group recently developed a picture stimulus set intended to depict experiences (e.g., interpersonal violations) that are relevant to BPD patients (Sloan et al., 2010). The stimulus set was developed to use in investigations of emotional responding with BPD participants. Unfortunately, the stimulus set was developed while the study described here was underway. We hope that future studies will investigate whether group differences in emotional responding are observed when using emotion-eliciting stimuli that are BPD-relevant.

Another issue concerns the narrow band of methods used to examine emotional responding. Different assessment methods may produce different findings. A multi-method, multi-trait evaluation of emotional dysregulation would facilitate our understanding of how affect dysregulation may vary as a function of the method used to index it.

The laboratory setting may have also influenced our ability to observe emotion dysregulation thought to be central to BPD. Investigating emotional responding of BPD individuals outside of the lab may allow for a more accurate observation of BPD emotional responding in everyday life. Another possible explanation for the lack of group differences in emotional responding obtained here and by others (Herpertz et al., 1999, 2000, 2001) is that the emotion difficulties in BPD may not be found in all individuals with BPD. Indeed, a 
diagnosis of BPD can be obtained without displaying symptoms of emotion dysregulation (APA, 1994) It is possible that only a subset of BPD individuals display emotion regulation difficulties.

Although there are a number of strengths of this study, there are limitations that should be considered. First, the sample size was relatively small, although similar to that used by Herpertz and colleagues (1999, 2000, 2001). The effect size of emotion regulation difficulties in BPD may be too small to have been detected with the sample size included in our study. The present study also included only women, which may limit the generalizability of our findings. However, the majority of individuals diagnosed with BPD are women (APA, 1994).

Taken together, laboratory studies examining emotional responding in BPD have not documented the emotion dysregulation that is thought to be a core feature of the disorder and has been observed in studies using self-report measures (see Rosenthal et al., 2008, for a review). Future studies should consider using BPD-relevant stimuli, using multiple measures of emotional responding, and study emotional responding outside of a controlled-laboratory environment.

\section{Acknowledgments}

This study was supported by a National Institute of Mental Health grant (R21MH069444-03) awarded to Brett T. Litz.

\section{References}

American Psychiatric Association. Diagnostic and statistical manual of mental disorders. 4th ed.. Author; Washington, DC: 1994.

Beck, AT.; Freeman, A. Cognitive Therapy of Personality Disorders. Guilford Press; New York: 1990.

Bradley MM, Codispoti M, Cuthbert BN, Lang PJ. Emotion and motivation I: Defensive and appetitive reactions in picture processing. Emotion. 2001; 1:276-298. DOI: 10.1037/1528-3542.1.3.276. [PubMed: 12934687]

Bradley MM, Lang PJ. Measuring emotion: The Self-Assessment Manikin and the semantic differential. Journal of Behavior Therapy and Experimental Psychiatry. 1994; 25:49-59. DOI: 10.1016/0005-7916(94)90063-9. [PubMed: 7962581]

Cohen, J. Statistical power analysis for the behavioral sciences. 2nd ed.. Lawrence Erlbaum; Hillsdale, NJ: 1988.

Ebner, Primer U, Schlotz W, Kleindienst N, Rosenthal MZ, Detterer L, Linehan M, Bohus M. Distress and affective dysregulation in patients with borderline personality disorder: A psychophysiologial ambulatory monitoring study. Journal of Nervous and Mental Disease. 2008; 196:314-320. DOI: 10.1097/NMD.0b013e31816a493f. [PubMed: 18414126]

First, MB.; Spitzer, RL.; Gibbon, M.; Williams, J. Structured Clinical Interview for DSM-IV Axis I Disorders - Patient Edition (SCID-I/P, Version 2.0). Biometrics Research Department, New York State Psychiatric Institute; New York: 1996.

Fridlund AJ, Cacioppo JT. Guidelines for human electromyographic research. Psychophysiology. 1986; 23:567-589. DOI: 10.1111/j.1469-8986.1986.tb00676.x. [PubMed: 3809364]

Hazlett EA, Speiser LJ, Goodman M, Roy M, Carrizal M, Wynn JK, et al. Exaggerated affectmodulated startle during unpleasant stimuli in borderline personality disorder. Biological Psychiatry. 2007; 62(3):250-255. doi:10.1016/j.biopsych.2006.10.028. [PubMed: 17258691]

Herpertz SC, Dietrich TM, Wenning B, Krings T, Erberich SG, Willmes K, et al. Evidence of abnormal amygdala functioning in borderline personality disorder: A functional MRI study. 
Biological Psychiatry. 2001; 50:292-298. DOI: 10.1016/S0006-3223(01)01075-7. [PubMed: 11522264]

Herpertz SC, Kunert HJ, Schwenger UB, Sass H. Affective responsiveness in borderline personality disorder. A psychophysiological approach. American Journal of Psychiatry. 1999; 156:1550-1556. [PubMed: 10518165]

Herpertz SC, Schwenger UB, Kunert HJ, Lukas G, Gretzer U, Nutzmann J, Sass H. Emotional responses in patients with borderline as compared with avoidant personality disorder. Journal of Personality Disorders. 2000; 14(4):339-351. [PubMed: 11204341]

Hochhausen NM, Lorenz AR, Newman JP. Specifying the impulsivity of female inmates with borderline personality disorder. Journal of Abnormal Psychology. 2002; 111:495-501. DOI: 10.1037/0021-843X.111.3.495. [PubMed: 12150425]

Keller MB, Lavori PW, Friedman B, Nielsen E. The longitudinal interval follow-up evaluation: A comprehensive method for assessing outcome in prospective longitudinal studies. Archives of General Psychiatry. 1987; 44(6):540-548. [PubMed: 3579500]

Kirk RE. Practical significance: A concept whose time has come. Educational and Psychological Measurement. 1996; 56(5):746-759. DOI:10.1177/0013164496056005002.

Lang, PJ.; Bradley, MM.; Cuthbert, BN. International affective picture system (IAPS): Affective ratings of pictures and instructional manual. University of Florida; Gainesville, FL: 2005. Technical Report A-6

Lang PJ, Cuthbert BN. Affective information processing and the assessment of anxiety. Journal of Behavioral Assessment. 1984; 6:376-395. DOI: 10.1007/BF01321326.

Linehan, MM. Cognitive-Behavioral Treatment of Borderline Personality Disorder. Guilford Press; New York: 1993.

Raudenbush, SW.; Bryk, AS. Hierarchical linear models: Applications and data analysis methods. 2nd ed.. Sage; Thousand Oaks, CA: 2002.

Raudenbush, S.; Bryk, A.; Congdon, R. HLM 6: Hierarchical linear and nonlinear modeling [Computer software and manual]. Scientific Software International; Lincolnwood, IL: 2005.

Rosenthal MZ, Gratz K, Kosson DS, Cheavens JS, Lejuez CW, Lynch TR. Borderline personality disorder and emotional responding. A review of the research literature. Clinical Psychology Review. 2008; 28:75-91. DOI: 10.1016/j.cpr.2007.04.001. [PubMed: 17544188]

Sloan DM, Sege CT, McSweeney LB, Suvak MK, Shea MT, Litz BT. Development of a borderline personality disorder-relevant picture stimulus set. Journal of Personality Disorders. 2010; 24:664675. DOI: 10.1521/pedi.2010.24.5.664. [PubMed: 20958174]

Zanarini MC, Skodol AE, Bender D, Dolan R, Sanislow C, Schaefer E, et al. The Collaborative Longitudinal Personality Disorders Study: Reliability of Axis I and II diagnoses. Journal of Personality Disorders. 2000; 14(4):291-299. [PubMed: 11213787]

Zanarini, MC.; Frankenburg, FR.; Sickel, AE.; Yong, L. The Diagnostic Interview for DSM-IV Personality Disorders (DIPD-IV). McLean Hospital; Boston, MA: 1996. 


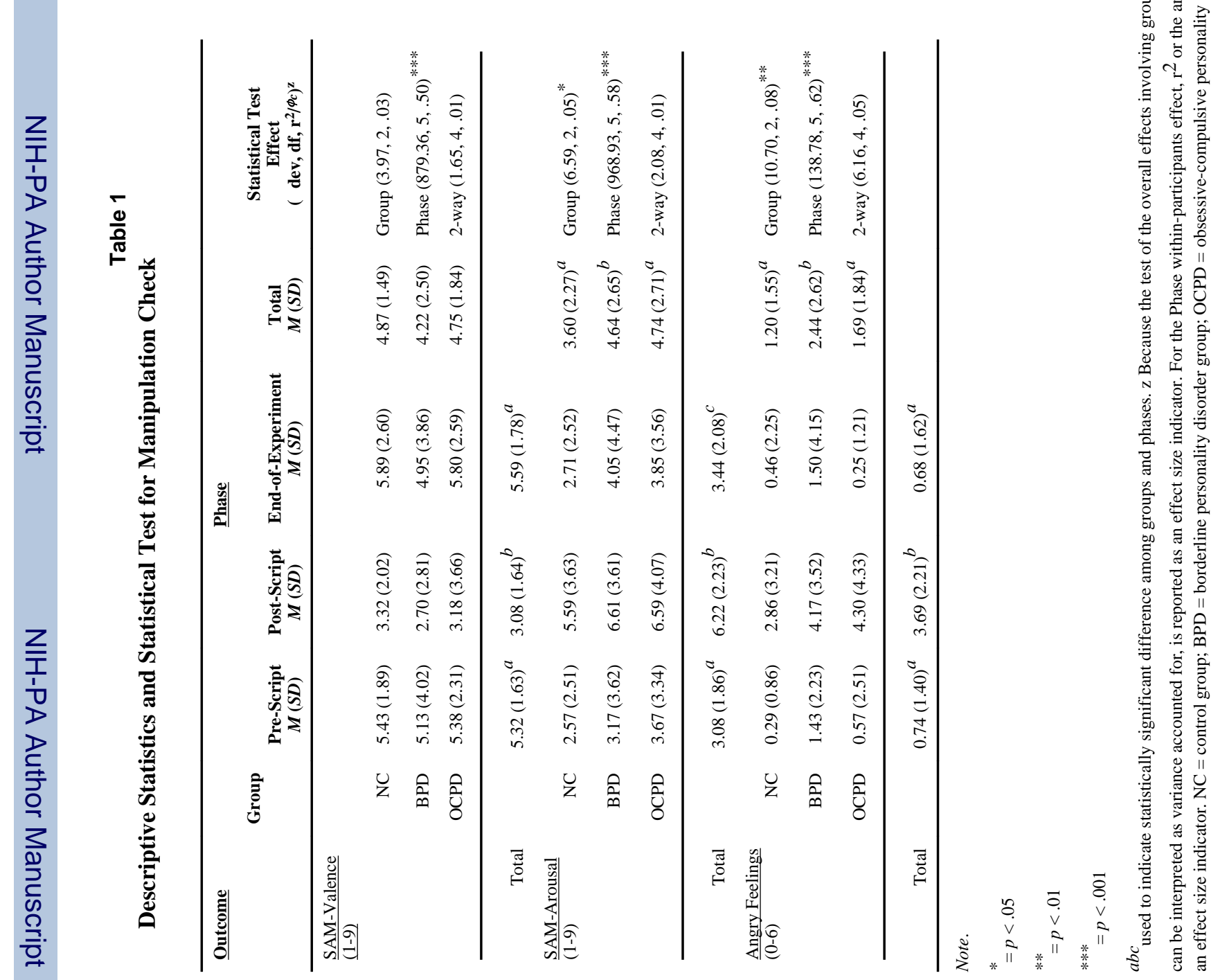




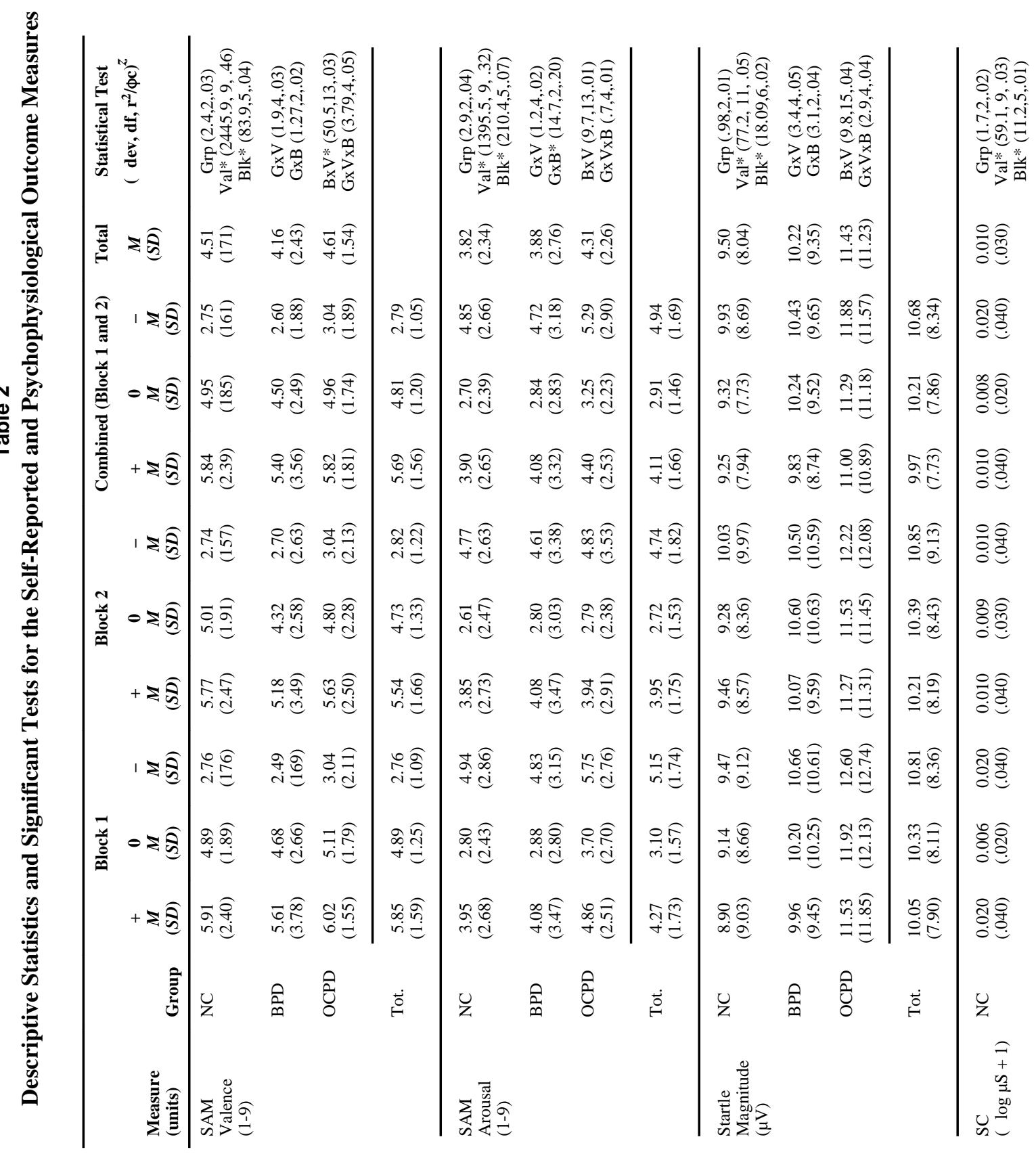




\begin{tabular}{|c|c|c|c|c|c|c|c|c|c|c|c|c|}
\hline 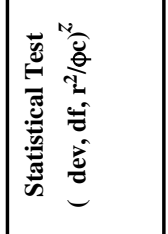 & 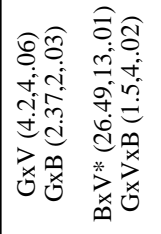 & & 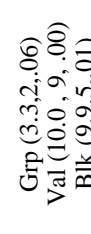 & 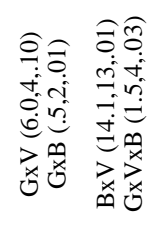 & & 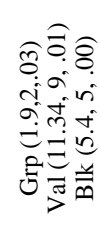 & 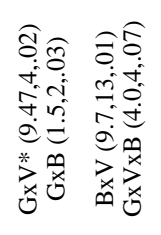 & & 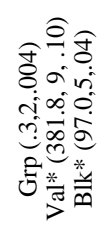 & 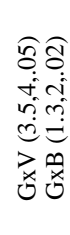 & 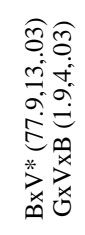 & \\
\hline 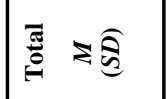 & : & & ণิ & 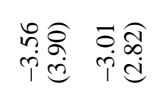 & & 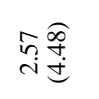 & 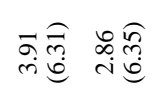 & & $\stackrel{d}{0} \cdot \hat{g}$ & $\stackrel{\text { so }}{\circ}$ & to & \\
\hline 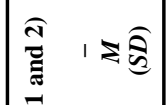 & 응 & 응 & 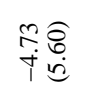 & 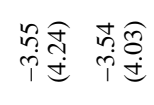 & $\begin{array}{l}\text { 워 } \\
\text { id }\end{array}$ & $\stackrel{\text { m. }}{\widehat{\widehat{o}}+\dot{+}}$ & 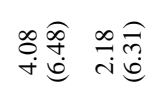 & 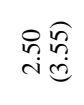 & : & $\stackrel{\leftrightarrow}{:}$ & $\stackrel{8}{0}$ & 웜 \\
\hline 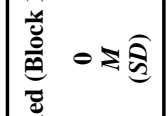 & 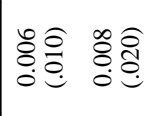 & 串 & 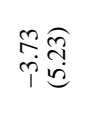 & 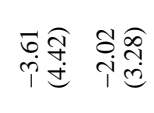 & 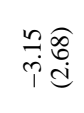 & ๙ָণ & ત્રે & 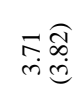 & ¿̊. & $\stackrel{\text { o̊ }}{\circ}$ & $\stackrel{\tilde{o}}{0} \widehat{\partial}$ & : \\
\hline 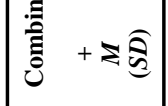 & 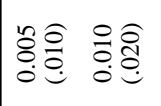 & 응 & 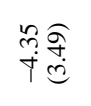 & 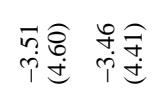 & $\underset{i}{i d}$ & †엉 & 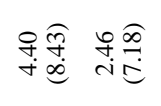 & 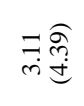 & 家昏 & ¿ & o̊ & 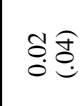 \\
\hline 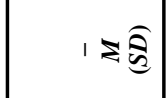 & 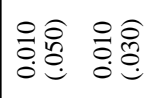 & 응 & 孚哥 & 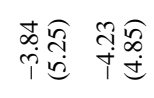 & 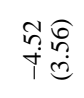 & 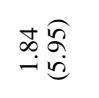 & 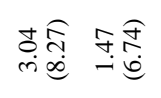 & 공 & 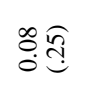 & 苞 & $\stackrel{8}{0} \stackrel{?}{]}$ & $\stackrel{8}{:}$ \\
\hline 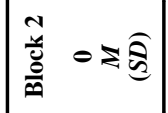 & 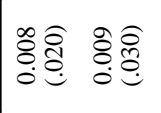 & 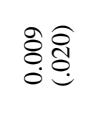 & 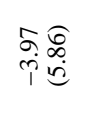 & 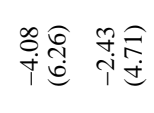 & 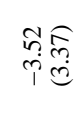 & तi & 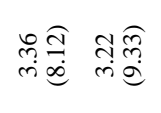 & 赵 & s̊. & $\stackrel{8}{0}$ & o̊ & 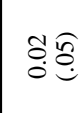 \\
\hline$+\Sigma \widehat{\widehat{C}}$ & : & 응 & 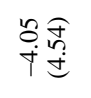 & 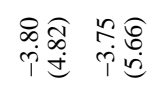 & $\begin{array}{l}\hat{\infty} \widehat{\infty} \\
i \stackrel{\infty}{i} \\
i \stackrel{d}{d}\end{array}$ & 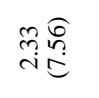 & 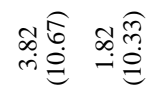 & 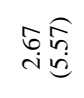 & s̊d & $\overline{0}$ & o̊ & 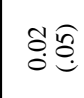 \\
\hline $1 \Sigma \widehat{\widehat{C}}$ & 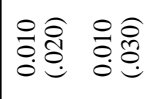 & 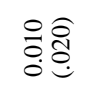 & 孛㠻 & 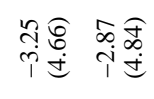 & 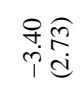 & 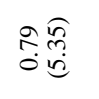 & 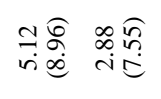 & 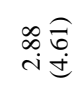 & : & $\stackrel{8}{\circ}$ & : & $\stackrel{\circ}{\circ}$ \\
\hline 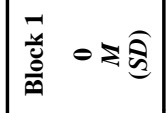 & 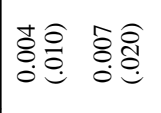 & 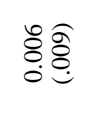 & 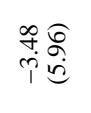 & 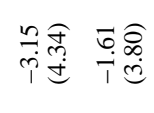 & $\stackrel{\infty}{i} \underset{\hat{i}}{i}$ & 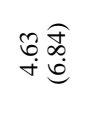 & $\begin{array}{ll} \pm \widehat{\partial} & \stackrel{\partial}{\sigma} \\
\dot{m} \dot{\infty} & \dot{\sigma}\end{array}$ & 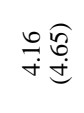 & ¿̊. & $\stackrel{+}{\circ}$ & 导 & $\stackrel{8}{\&}$ \\
\hline$+\Sigma \widehat{\widehat{C}}$ & 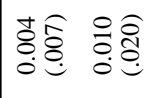 & 응 & 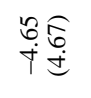 & 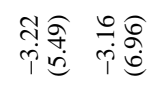 & $\begin{array}{l}\text { Re } \\
\text { iç }\end{array}$ & 웜 & 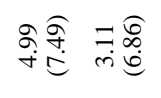 & 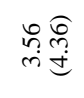 & 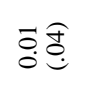 & 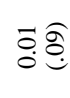 & ชิత & $\overline{0}$ \\
\hline 产 & 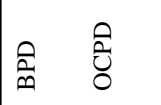 & $\stackrel{\dot{0}}{\ominus}$ & $\mathrm{z}$ & ڤે & $\dot{ث}$ & z & 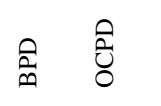 & $\stackrel{\dot{\bullet}}{\bullet}$ & 乙 & 㝕 & : & $\stackrel{\dot{0}}{\circ}$ \\
\hline 竧绨 & & & 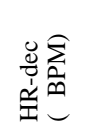 & & & 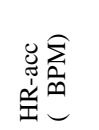 & & & 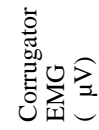 & & & \\
\hline
\end{tabular}




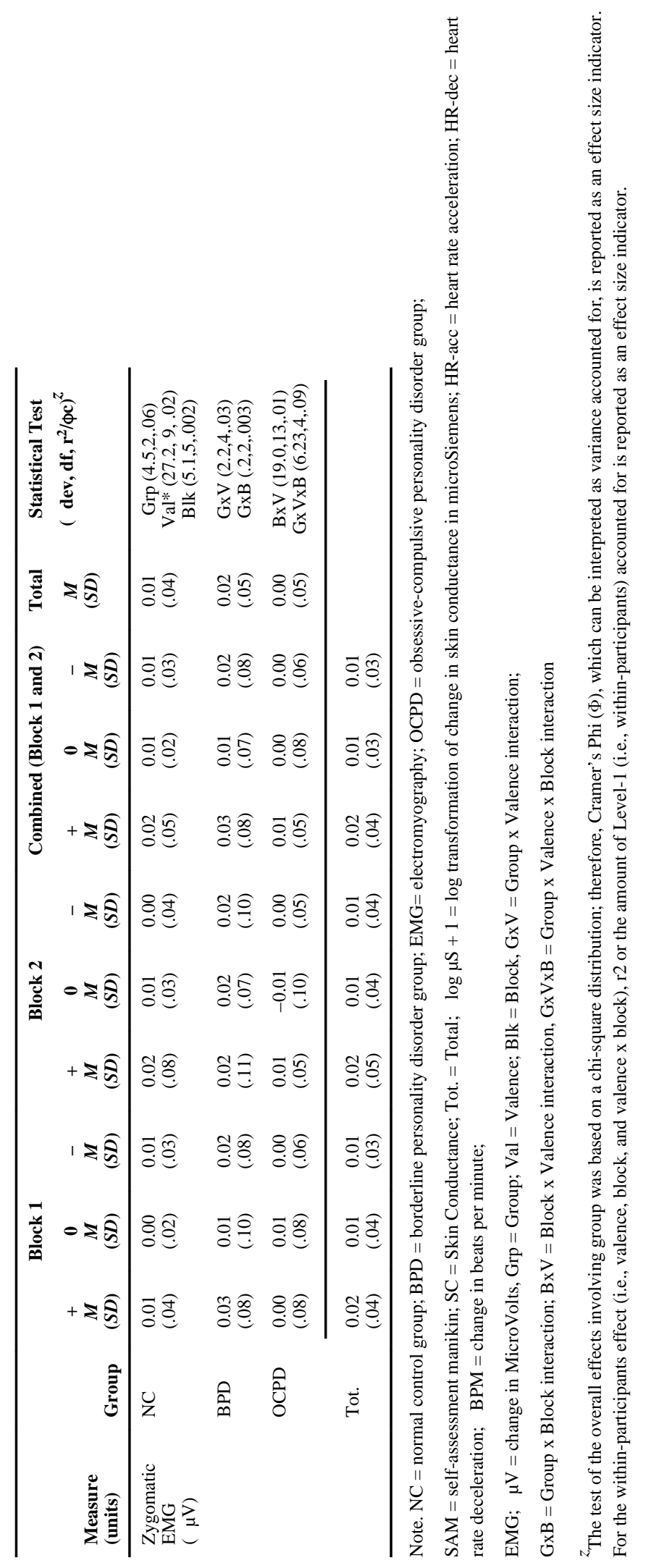

